'There are, of course, very many cases of nasal sinusitis in which the lacrymal sac is not affected, at least, not so grossly affected as to draw the patient's or surgeon's attention to it; but, to make an analogy, one must remember the small proportion of epididymitis in cases of gonorrhoea, or how seldom the parotids are affected in oral sepsis. The fact that the nasal duct opens into the inferior meatus high up under cover of the inferior turbinal, and its peculiar direction from the eye-nawely, downwards, outwards, and backwards into the nosewhich provides good drainage even in the recumbent posture, may be factors in its immunity from contamination in all cases of nasal sinusitis.

In this case the prevailing organism in culture from the antrum pus and the lacrymal sac pus was the pneumococcus, which is often met with in lacrymal sac affections. Provided the nasal duct is fairly patent-and there are many cases of dacryocystitis, and certainly of commencing epiphora, where this is so-and nasal sinus disease is found to be present, the removal of the latter can reasonably be expected to cure or improve the eye condition. If there is a definite stricture of the duct, then, of course, one must resort to other measures-removal of the sac, or intranasal drainage of the tears.

I have operated on three other cases of a similar nature (dacryocystitis and antrum disease), but nothing like a cure of the dacryocystitis was obtained in any of them, owing to the fact that the nasal duct was either completely or very much obstructed to commence with. Incidentally, a case like the above (also Case rv) helps one to leave, at times, the somewhat narrow grooves of routine ophthalmic work, and to remember that many ophthalmic conditions are merely symptoms of disease of a neighbouring organ, or of a general infection.

With regard to Cases II and III, the relationship of nasal sinus disease to otitis media is well known. It is difficult t) be always sure which process commences first, but nitimately a vicious circle is established, and it is impor tant to correct any existent nasal disease before undertaking the mastoid operation, in order to avoid causing clisappointment to the patient, who expects a dry ear within a reasonable time after the operation, certainly within six monthis.

Case III shows the excellent result on the ear disease of attending to nasal sinusitis first; but the chief poin revarding Cases III and IV is the age of the patients-12 and 15 years. I find that antrum disease is by no means uncommon in children about this age, or even younger, and a nasal discharge at this time of life is often, and correctly. put down to the presence of adenuids; but if the nasa discharge does not cease within two months of the adenoir operation, a careful examination of the nose ivill frequently reveal the cause of the trouble in the presence of a deviated septum, or antrum disease, or both combined.

\section{Method of Operation.}

This is not the place to discuss the merits of the intranasal and the radical antrum operations. Personally, I al ways employ the radical method, for many reasons.

As regards the type of operation, the Caldwell-Luc was done in all these cases, the technique employed being that of Jr. W. S. Syme of Glasgow. By this method a better view is obtained of the interior of the antrum, and the vertical incision has many other obvious advantages; moreover, the whole operation can be done with com parative comfort to the patient under local anaesthesia, preceded by a hypodermic of morphine or omnopon. 'The great advantage of the method is that it is practically bloodless, whether done under local or general anaesthesia.

C. H. Manlove (Philippine Journ. Sci., Manila, 1917, xii, Sec. B, 149-163), who records two cases with necropsies, finds that intestinal infection with Balantidium coli is not infrequent in the Philippine Islands, and that among 68 cases there were 7 deaths. Associated with Balan. tidium coli there may be catarrhal inflammation or deep burrowing ulceration, and macroscopically these lesions are indistinguishable from those in amoebic dysentery. Microscopically the cellular changes are the same, but the causal organisms can be recognized. In Manlove's cases eosinophilia of the intestinal wall, though emphasized by previous writers, was not prominent. Any part of the colon may be affected. As in amoebiasis, extensive intestinal lesions may be present in balantidiasis without causing symptoms.

\section{INTRAVENOUS SALINE IN BLACKWATER FEVER.}

By ADAM PATRICK, M.D., Captain R.A.M.C.(T).

AN attack of blackwater fever begins often with a rapid rise of temperature, malaise, and sometimes rigor and vomiting. The urine becomes of a port-wine or darker red colour, and shows when collected a copious brown granular precipitate. The amount of urine passed quickly falls off, and within forty-eight hours the symptoms may resemble those of uraemia, with vomiting, headache, and oliguria. 'The patient's prospects of recorery are to bo judged to some extent by the amount of urine passed, and if only a few ounces are secreted in twenty-four hours thu outlook is distinctly bad. There is ground for believin: that the decrease in the urinary secretion is partly to be accounted for by the deposit of the brown amorphous material in the kidney tubules in such quantity as to obstruct them, or even to block them completely. The febrile condition increases the concentration of the urine, and decrease in the awount of water passing through the kidneys favours the silting-up process in the tubules. Support is given to this view by the finding of brown tube casts in the first specimens of urine passed after the attack begins. These are not of the nature of the caste found in nephritis, but are composed of the brown material excreter with the urine. Their shape shows that they have been formed in the tubales, and it is not unreasonable to suppose that if little water passes through the kidney they may not be washed ont at all, but remain in the tubules and block them. If this view is correct one of the most important lines of treatment is to increase diuresis, and the speediest method of doing this is to inject a sorlium chloride solution intravenously. This was tried in the foliowing case, and probably saved the patient's life.

J. II., ager 26, had his first attack of subtertian malaria in Iracedonia in June, 1917, and suffered from it pretty constantiy for the next six months, spending nearly all his time in hos pital. He was transferred to a Malta lospital in December. 1917, and when admitted there on Decembe 26 th 1917, and when admitted there on December 26 th was pale and cachectic. Subtertian parasites (crescents) were found in the . He had no prrexia, and no quinine was given to him.

On fanuary $12 \mathrm{th}, 1918$, he began to feel ont of sorts, and on January 13th his temperatire was a little elevated. A single lose of quinine sulphate (10 grains) was given by the month. In the evening ho was shivery, and during the night passed red urine. Next morning (January 14th) the temperature was $104^{\circ} \mathrm{F}$, and a blood film showed a few subtertian rings. The urine was red, with a copions brown deposit, but withont red blood corpuscles. It was diminished in amount, though not markelly. In the evening the temperatnre fell to 102.6

Next day (Jannary 15th) the highest tempe:ature was 100.20 but his condition was much worse, and in the evening it looked as though he were going to die. The pulse-rate was 122, in association with a temperature of $100^{\circ}$; the urine had become very scanty and dark red in colour ; he vomited frequenily and very scanty and dark red in co!our ; he vomited frequenty and complained of headache. At 10.30 p.m. 1,500 c.cm. Of stenlized tap-water, with sodium chloride (1 per cent.) in solution, was injected intravenously. Immediately before this, at the sug gestion of Colonel Sir A. Garrod, A.M.S., cups were put on over the kianeys. Within one and a half hours the patient had passed urine which was lighter in colour, and within the twelve hours following the injection he passed $1,100 \mathrm{c.cm}$. Next morning he was pale and yellowisb, but felt much better, ani no longer looked like a man who was likely to die. By 5 p.m. of the same day fonnary 16 th haemoglobin had disappeared from the urine, and lie made a rapid and uninterrupted recovery.

The importance of increasing the flow of urine in such cases is urged by the textbooks on tropical medicine, but they generally suggest giving saline solution intracellularly or by the rectum. ${ }^{1}$ Evidently what is wanted is a rapid copious passage of water through the renal channels, and this is better obtained by an intravenous than by either a subcutaneous or a rectal injection. It is generally recog. nized that water injected directly into the circulation is rapidly excreted. 'This is a disadvantage if the object of the injection is to raise the blood pressure, but it is just what is wanted in a case of blackwater fever such as that just described. Once the tubules become blocked, attempts to promote diuresis may not avail, and tlierefore it would seem advisable to give the intravenous injection, or injections, before the patient reaches this dangerous stage, TCastellani and Chalmera, Manical, of Tropical Medicine (19ił),
p. 922 ; Manson, Tropical Diseabes (1914), 300 . 